\title{
Una actuación de enfermería: intentar aliviar el dolor en las punciones de hemodiálisis
}

\author{
Isabel Granados Navarrete* \\ Dolores Abril Sabater* \\ Fina Alcaraz Busqueta* \\ Núria Mañé Buixo* \\ Judith Padilla Ruiz* \\ Jordi Real Gatius* * \\ Manel Solano Pallarés* \\ Elios Yuste Giménez*
}

\author{
* Servicio de Nefrología. \\ **Servicio de Epidemiología \\ Corporación Sanitaria Parc Taulí \\ Sabadell. Barcelona
}

\section{RESUMEN}

La teoría de control de la compuerta, propuesta originalmente en 1965 (Melzack y Wall), sugiere que la estimulación cutánea puede aliviar el dolor mediante la activación de las fibras nerviosas nociceptivas de diámetro grande.

Nuestro objetivo consiste en averiguar si, en la práctica clínica, un dolor breve- moderado puede aliviar un dolor más intenso en una zona cercana, en este caso la punción en hemodiálisis.

La técnica consiste en realizar una hiperestimulación sensorial mediante la mayor compresión de smarch utilizado para la punción, para posteriormente recoger la valoración del grado de dolor por parte del paciente.

Podemos concluir que la compresión extra del miembro portador del acceso vascular reduce significativamente el grado de dolor en las punciones venosas. La compresión extra en las punciones arteriales también reduce el grado de dolor, aunque no hemos encontrado diferencias significativas.

Correspondencia:

Isabel Granados Navarrete

Servicio de Nefrología

Corporación Sanitaria Parc Taulí

$C /$ Parc Taulí s/n

08208 Sabadell. Barcelona
PALABRAS CLAVE: FÍSTULA ARTERIOVENOSA

DOLOR

TEORÍA DEL CONTROL DE LA COMPUERTA HIPERESTIMULACIÓN CUTÁNEA

HEMODIÁLISIS

\section{A NURSING ACTION: TRYING TO ALLEVIATE PAIN IN HAEMODIALYSIS PUNCTURES}

\section{SUMMARY}

The gate control theory, originally proposed in 1965 (Melzack and Wall), suggests that cutaneous stimulation can alleviate Pain by activating large-diameter nociceptive nerve fibres.

Our aim is to verify whether, in clinical practice, a brief-moderate pain can alleviate more intense pain in a nearby area, in this case the haemodialysis puncture.

The technique consists of creating sensorial hyperstimulation by greater compression of the Esmarch used for the puncture, and then examining the evaluation of the level of pain by the patient.

We can conclude that the extra compression of the member bearing the vascular access significantly reduces the level of pain in venous punctures. The extra compression in arterial punctures also reduces the level of pain, although we have not found significant differences. 


\section{KEY WORDS: ARTERIOVENOUS FISTULA \\ PAIN \\ GATE CONTROL THEORY \\ CUTANEOUS HYPERSTIMULATION \\ HAEMODIALYSIS}

puede explicar que esto alivia el dolor de las contracciones, así como un hombre que se da palmadas en la cara tras aplicarse un tónico después del afeitado. El dolor breve-moderado parece producir impulsos nerviosos que actúan cono un complejo mecanismo inhibidor neural que conduce a un alivio de un dolor más intenso y prolongado en una zona cercana o distante. Existen en la bibliografía técnicas demostradas que alivian el dolor de una inyección intramuscular en el músculo deltoides, mediante el pellizco enérgico del citado músculo (Locsin, 1985) y que consiste en:

\section{INTRODUCCIÓN}

El enfermo en tratamiento sustitutivo renal acude a nuestro centro tres veces por semana, pasando en él alrededor de 4-5 horas. En cada sesión recibe, en el caso de pacientes con bipuntura, dos punciones con agujas del calibre 15-16 G. La opción del trasplante en ocasiones no es factible y, en estos casos, nos encontramos con personas jóvenes, de alrededor 50-60 años que tienen en la hemodiálisis su única forma de supervivencia.

En todos nuestros pacientes, pero en especial para éstos, la punción es muy importante, no sólo para una buena calidad de diálisis, sino por lo que esta experiencia "traumática" supone para el paciente. Además, está demostrado que la experiencia repetida o prolongada del dolor suele disminuir la tolerancia a éste. Actualmente la población portadora de fístula arterio-venosa (FAVI) es de 100 pacientes, lo que supone un $79 \%$ del total de pacientes en programa de hemodiálisis.

La teoría de control de la compuerta, propuesta originalmente en 1965 (Melzack y Wall), nos dice que:

- La estimulación cutánea puede aliviar el dolor: La activación de las fibras nerviosas de diámetro grueso alivia el dolor. Puesto que la piel está ricamente dotada de estas fibras, muchos tipos de estimulación táctil tienen el potencial de alivio del dolor.

- Una estimulación sensorial "normal" o excesiva puede aliviar el dolor. El sistema reticular del tronco del encéfalo puede inhibir los estímulos que le llegan si la persona está recibiendo un estímulo sensorial suficiente o excesivo.

En un sentido amplio, esta teoría sugiere que la estimulación de la piel puede activar las fibras de diámetro grande. A su vez, esta activación provocará una inhibición de los mensajes dolorosos transportados por las fibras más pequeñas, es decir, cierran la compuerta a la transmisión de impulsos sentidos como dolorosos.

La analgesia por hiperestimulación o contrairritación es un tipo de estimulación cutánea. Obviamente, a niveles intensos, estos estímulos son con frecuencia dolorosos. Una mujer que se muerde los labios durante las contracciones del parto

\section{Técnica de pellizcar-agarrar para la inyección intra-} muscular en el músculo deltoides

1. Agarrar el músculo deltoides con el dedo pulgar por un lado y con los restantes dedos de la mano por el otro.

2. Tirar hacia Ud. del músculo deltoides aproximadamente entre 3,5 y $4 \mathrm{~cm}$.

3. Apretar pellizcando el músculo, ejerciendo la presión suficiente para provocar algún malestar, haciendo que el músculo sobresalga y la piel se tense.

4. Con la otra mano administrar la inyección, en un ángulo de $90^{\circ}$, insertando la aguja aproximadamente $4 \mathrm{~cm}$ en el músculo deltoides.

5. Continuar cogiendo-pellizcando el músculo hasta retirar la aguja y se presione una torunda de algodón sobre el lugar de inyección.

6. Con la torunda de algodón frotar la zona con ligera presión durante 5 a 10 seg.

\section{OBJETIVOS}

\section{Objetivo general}

- Averiguar si en la práctica clínica, un dolor breve-moderado puede aliviar un dolor más intenso en una zona cercana, en este caso la punción en hemodiálisis.

\section{Objetivos específicos}

- Disminuir la ansiedad relacionada con las punciones

- Minimizar las reacciones vaso-vagales relacionadas con el dolor en las punciones.

\section{MATERIAL Y MÉTODOS}

El trabajo fue realizado en la Unidad de Hemodiálisis de la Corporació Sanitària Parc Taulí, Sabadell (Barcelona). Los pacientes se seleccionaron entre los 126 pacientes en tratamiento sustitutivo renal mediante hemodiálisis de la Corporació Sanitària Parc Taulí, según los siguientes criterios de inclusión: 1) Portador de fístula arterio-venosa autóloga funcionante; 2) Diálisis por medio de bipuntura; 3) Capacidad 
cognitiva conservada; $y, 4)$ No utilización de otros métodos para el alivio del dolor. Inicialmente se pasó una hoja informativa a los pacientes, pidiendo su colaboración en el estudio, entre los que fueron considerados óptimos de acuerdo a los criterios de inclusión.

Estudio prospectivo, de 9 meses de duración, en el cual se valoró el grado de dolor percibido antes y después de la aplicación de la técnica estudiada. Incluye a 32 pacientes, 11 mujeres y 21 hombres, con un promedio de edad de 58 años, entre un rango de 28 a 87 años. De éstos, un 50\% son portadores de FAVI radial, mientras el otro 50\% la llevan en el codo. Nos encontramos ante una población con accesos vasculares "jóvenes", ya que un 59,4\% son portadores de FAVIs con antigüedad inferior a los tres años. Se valoró el dolor percibido por el paciente usando la escala del dolor EVA, que es una escala numérica, que va del 0 al 10, en la cual el 0 es ausencia de dolor y el 10 es el dolor más intenso que se puede sufrir. En ella el paciente indicará, numéricamente, su percepción del dolor, con y sin la aplicación de la técnica.

La técnica consiste en realizar una hiperestimulación sensorial mediante la mayor compresión de smarch utilizado para la punción. La utilización de smarchs "milimétricos" (figura 1) nos permite realizar una compresión objetiva y repetible en cada paciente. La compresión de la extremidad se realiza de forma que produzca cierta molestia al paciente, pero siempre evitando un compromiso en la irrigación del miembro. El nivel "óptimo" de compresión, se valora mediante un consenso entre el paciente y la enfermera/o que realiza la técnica. El paciente indica en qué momento siente molestia con la compresión, y la enfermera controla que no exista compromiso en la irrigación del miembro. Es importante mencionar, que este tipo de smarch produce una compresión uniforme en todo el contorno del brazo, sin pellizcar y sin enrollarse, de forma que la pequeña molestia ocasionada no es en ningún momento dolorosa. Todas las punciones efectuadas a un mismo paciente fueron realizadas por el mismo profesional, de forma que se intenta minimizar variables que no podemos controlar como, por ejemplo, la destreza personal de cada individuo. Además, no se puncionó durante el estudio zonas vasculares nuevas o poco puncionadas, es decir, se utilizaron las zonas habituales de punción.

Para la valoración, se diseñó una hoja de recogida de datos personalizada al tipo de estudio que llevamos a cabo (anexo 1). Antes de empezar con el smarch milimétrico, se realizó una medición con el smarch habitual, con la finalidad de comparar a posteriori los dos tipos de smarchs y constituir una especie de "dolor basal" en los pacientes que participan en el estudio. Una vez hecho esto, las posteriores valoraciones fueron con el smarch milimétrico. Consecutivamente se realizaron las punciones, sin aplicar la técnica y aplicándola (figura 2), tras lo
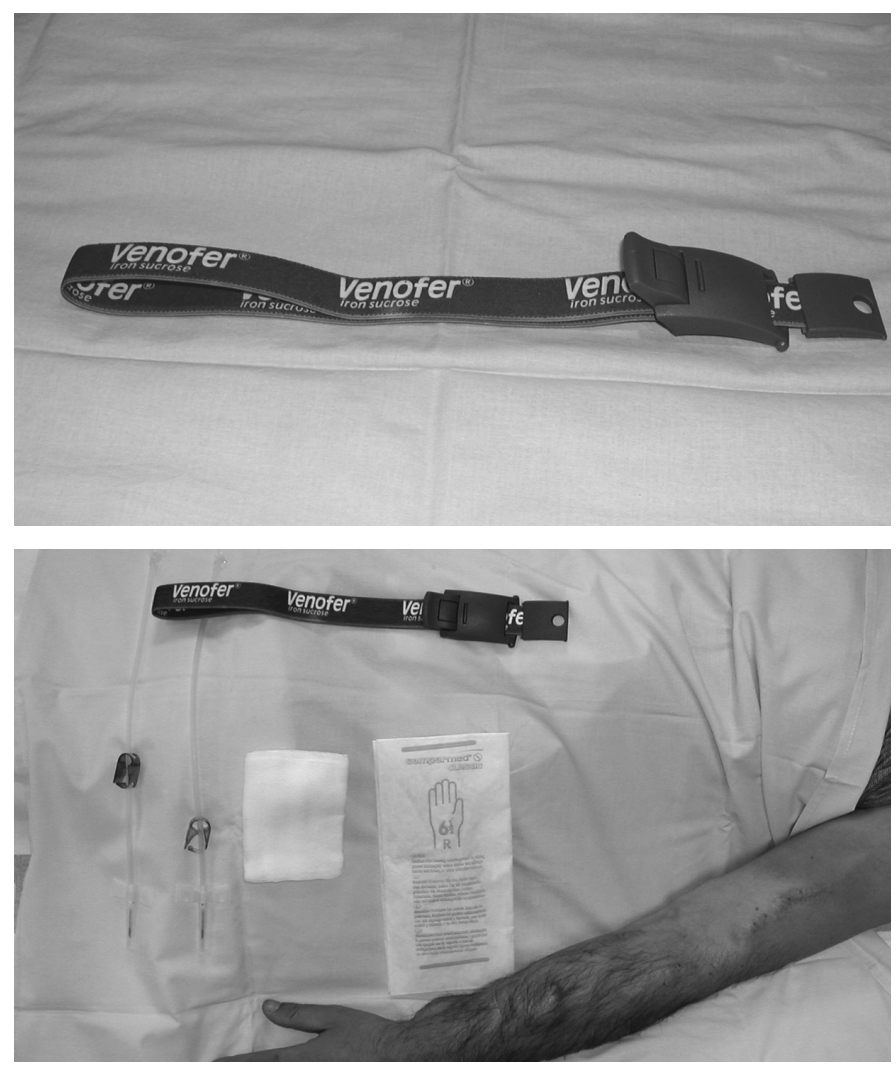

Figura 1. Smarch milimétrico y material utilizado para la punción.
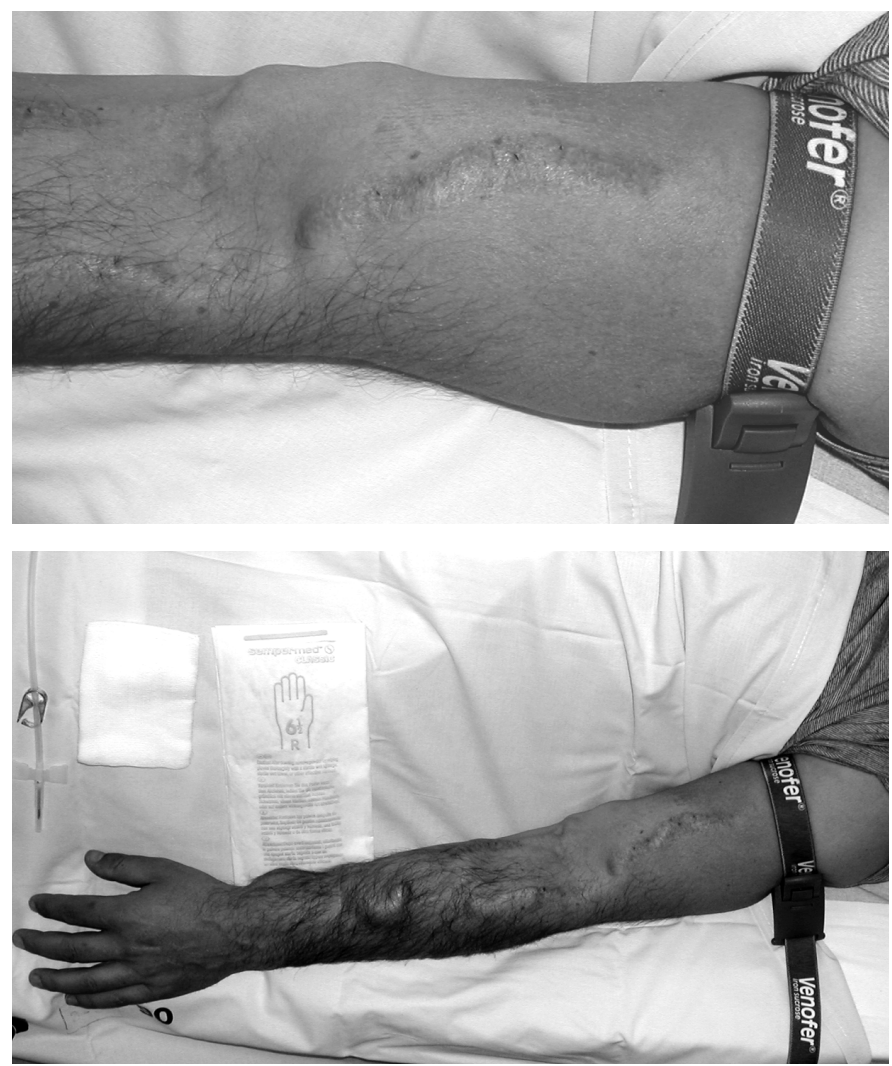

Figura 2. Aplicación de la técnica "compresión extra". 
cual fue recogida la valoración del grado de dolor por parte del paciente. Se realizaron medidas durante 10 sesiones de HD, en cada una de las cuales se valoró el dolor en arteria y en vena. Se alternaron en estas mediciones la aplicación de compresión "extra" y de compresión "normal", con la finalidad de descartar aspectos relacionados con el azar. Además, no todos los pacientes seguían el mismo orden. Por último, al finalizar la recogida de datos, se les pidió a los pacientes su opinión al respecto del trabajo realizado.

Los datos recogidos fueron analizados mediante un sistema informático con paquete estadístico SPSS10, en concreto tablas de frecuencias, T de Student para muestras relacionadas, análisis de la varianza y estudio de la correlación. Para esto, contamos con la colaboración del Servicio de Epidemiología del Hospital.

\section{RESULTADOS}

Una vez obtenidas todas las puntuaciones de las diferentes punciones realizadas a todos los pacientes, se realizó un estadístico descriptivo, el cual nos permite saber el promedio de dolor que sintieron los individuos sujetos a estudio (tabla1). Se puede observar que la media va disminuyendo, tanto en la punción arterial como en la venosa, al aplicar la compresión "extra" del smarch. En las punciones venosas, por ejemplo, si el promedio del dolor sentido por los pacientes es de 3'59 con el smarch habitual (en una escala de 0 a 10), desciende hasta sentir una media de 1,75 con la compresión "extra" del smarch "milimétrico". Es decir, observamos una gran disminución de la media si comparamos la utilización del smarch habitual con el smarch "milimétrico".

\begin{tabular}{|c|c|c|}
\hline & ARTERIALES & VENOSAS \\
\hline PREVIOS & $2^{\prime} 75 \pm 2^{\prime} 03$ & $3^{\prime} 78 \pm 2^{\prime} 7$ \\
\hline COMPRESIÓN NORMAL & $2^{\prime} 08 \pm 1^{\prime} 47$ & $2^{\prime} 30 \pm 1^{\prime} 75$ \\
\hline COMPRESIÓN EXTRA & $1^{\prime} 85 \pm 1^{\prime} 67$ & $1^{\prime} 75 \pm 1^{\prime} 61$ \\
\hline
\end{tabular}

Tabla 1. Medias obtenidas en las punciones.

Se observa, a medida que se realizan las diferentes punciones, tanto con la aplicación de la técnica como sin ella, que el paciente define más el índice de su dolor, quizás por comparación con las punciones anteriores, de forma que se produce una disminución de la media de dolor a medida que pasa el tiempo en todos los casos.

Posteriormente al estadístico descriptivo, realizamos comparaciones entre las medias para descubrir si las diferencias son estadísticamente significativas, obteniendo los resultados refleja- dos en la tabla 2. Podemos observar que la disminución en el grado de dolor percibido es estadísticamente significativa en los siguientes pares:

- En todos los casos, si comparamos la utilización del smarch habitual con el milimétrico.

- En las punciones venosas, si comparamos la compresión normal de smarch milimétrico, con la compresión extra.

\begin{tabular}{|l|c|}
\hline Comparaciones & $\begin{array}{c}\text { Sig. } \\
\text { (bilateral) }\end{array}$ \\
\hline NORMAL.ART - EXTRA.ART & 0,094 \\
\hline NORMAL.VEN - EXTRA.VEN & 0,032 \\
\hline PREVIO.ART - NORMAL.ART & 0,045 \\
\hline PREVIO.ART - EXTRA.ART & 0,015 \\
\hline PREVIO.VEN - NORMAL.VEN & 0,000 \\
\hline PREVIO.VEN - EXTRA.VEN & 0,000 \\
\hline
\end{tabular}

Tabla 2. Prueba T Student para muestras relacionadas.

En todos los casos, los datos están correlacionados significativamente (tabla 3).

\begin{tabular}{|l|c|c|}
\hline & Correlación & Sig \\
\hline NORMAL.ART Y EXTRA.ART &, 894 &, 000 \\
\hline NORMAL.VEN Y EXTRA.VEN &, 833 &, 000 \\
\hline PREVIO.ART Y EXTRA.ART &, 448 &, 010 \\
\hline PREVIO.ART Y NORMAL.ART &, 503 &, 003 \\
\hline PREVIO.VEN Y EXTRA.VEN &, 654 &, 000 \\
\hline PREVIO.VEN Y NORMAL.VEN &, 733 &, 000 \\
\hline
\end{tabular}

Tabla 3. Correlaciones de muestras relacionadas.

La diferencia entre el dolor venoso con compresión normal y el dolor venoso con compresión extra es estadísticamente significativa ( $p=0.032$ ). Es decir, la compresión extra del miembro portador del acceso reduce significativamente el grado de dolor en las punciones venosas.

\section{DISCUSIÓN}

De un modo empírico, el ser humano ha utilizado desde la antigüedad la estimulación cutánea como un método para el alivio del dolor, por ejemplo frotándose la piel después de un golpe, o acariciándose el abdomen ante un dolor en esa zona. Sin embargo, el estudio del dolor, la algología, está en pañales. Antes de la introducción de la teoría del control de la compuerta en 1965 por Melzack y Wall había muy poco material en la literatura profesional sobre el tema del dolor. En relación al dolor en las punciones existen muchos trabajos descritos en 
la bibliografía estudiando los beneficios del uso de pomadas anestésicas tipo EMLA, cloretilo o, más recientemente, el uso de agujas congeladas. Sin embargo, el aprovechamiento de la anatomo-fisiología del sistema nervioso nociceptivo, está muy poco estudiado. Prueba de ello es la poca bibliografía encontrada sobre el tema, tanto a nivel médico como enfermero. Los efectos de la estimulación cutánea son variables e impredecibles, pero, en general, la expectativa es que se eliminará el dolor o disminuirá su intensidad ante la estimulación. Algunos métodos de estimulación cutánea, incluido éste, tienen indudablemente efectos de distracción, además de activar mecanismos que reducen realmente la intensidad del dolor.

La técnica que hemos descrito es sencilla y fácil de poner en práctica, puede ser utilizada junto con otras técnicas para el alivio del dolor, lo que se podría decir que su utilización es casi universal, en todos aquellos pacientes portadores de fístula arterio-venosa autóloga.

Pese a que el rango de dolor percibido por nuestros pacientes es totalmente amplio, va desde el 0 al 10 en la escala EVA, las medias de dolor expuestas en las tablas anteriores son bastante bajas, teniendo en cuenta que estos pacientes no utilizan otras técnicas para el alivio del dolor, que se trata de personas con una media de edad joven (58 años), y que la mayoría son portadores de accesos de menos de 3 años.

Por último, es conveniente destacar que, en todos los casos, tanto por parte de los pacientes como del personal de enfer- mería, la utilización de este tipo de smarch ha sido valorada muy positivamente, debido a que realiza una compresión homogénea en todo el contorno del brazo.

\section{CONCLUSIONES}

- La compresión extra del miembro portador del acceso reduce significativamente el grado de dolor en las punciones venosas. La compresión extra en las punciones arteriales también reduce el grado de dolor, aunque no hemos encontrado diferencias significativas.

- En todos los casos, hubo una disminución estadísticamente significativa entre el grado de dolor percibido con el smarch habitual y el milimétrico.

- En general, los pacientes afirman que este tipo de smarch es más cómodo, puesto que realiza una compresión uniforme sin causar dolor.

\section{BIBLIOGRAFÍA}

- McCaffery M, Beebe A. Dolor, Manual clínico para la práctica de enfermería. Barcelona: Salvat; 1995.

- Guyton A, May J. Tratado de Fisiología médica. 9a edición. Madrid: Interamericana; 1996.

- Forth W, Martin E, Meter K. The Relief of Pain. Germany: Ed. Hoechst; 1990. 


\section{Anexo 1. Hoja de recogida de datos}

PACIENTE:

SEXO:

EDAD: $\quad 20-40$

TIPO FAVI: RADIAL

EDAD FAVI: $<1$ a
$\mathrm{H} \quad \mathrm{D}$

41-60

\section{CODIGO PACIENTE:}

$61-80>80$

CODO

3-5 a. $\quad>5$ a.

\begin{tabular}{|c|c|c|c|c|c|c|c|c|c|c|c|c|}
\hline \multirow[t]{2}{*}{ PREVIO } & ART & 0 & 1 & 2 & 3 & 4 & 5 & 6 & 7 & 8 & 9 & 10 \\
\hline & VEN & 0 & 1 & 2 & 3 & 4 & 5 & 6 & 7 & 8 & 9 & 10 \\
\hline
\end{tabular}

\begin{tabular}{|l|c|c|ccccccccccc|}
\hline \multirow{3}{*}{ FECHA: } & $\begin{array}{c}\text { COMP } \\
\text { NORMAL }\end{array}$ & ART & 0 & 1 & 2 & 3 & 4 & 5 & 6 & 7 & 8 & 9 & 10 \\
\cline { 2 - 9 } & $\begin{array}{c}\text { COMP } \\
\text { EXTRA }\end{array}$ & VEN & 0 & 1 & 2 & 3 & 4 & 5 & 6 & 7 & 8 & 9 & 10 \\
\hline
\end{tabular}

\begin{tabular}{|l|c|c|cccccccccccc|}
\hline FECHA: & $\begin{array}{c}\text { COMP } \\
\text { NORMAL }\end{array}$ & ART & 0 & 1 & 2 & 3 & 4 & 5 & 6 & 7 & 8 & 9 & 10 \\
\cline { 2 - 9 } & $\begin{array}{c}\text { COMP } \\
\text { EXTRA }\end{array}$ & VEN & 0 & 1 & 2 & 3 & 4 & 5 & 6 & 7 & 8 & 9 & 10 \\
\hline
\end{tabular}

\begin{tabular}{|l|c|l|llllllllllll|}
\hline \multirow{2}{*}{ FECHA: } & $\begin{array}{c}\text { COMP } \\
\text { NORMAL }\end{array}$ & ART & 0 & 1 & 2 & 3 & 4 & 5 & 6 & 7 & 8 & 9 & 10 \\
\cline { 2 - 8 } & $\begin{array}{c}\text { COMP } \\
\text { EXTRA }\end{array}$ & VEN & 0 & 1 & 2 & 3 & 4 & 5 & 6 & 7 & 8 & 9 & 10 \\
\hline
\end{tabular}

\begin{tabular}{|l|c|l|llllllllllll|}
\hline FECHA: & $\begin{array}{c}\text { COMP } \\
\text { NORMAL }\end{array}$ & ART & 0 & 1 & 2 & 3 & 4 & 5 & 6 & 7 & 8 & 9 & 10 \\
\cline { 2 - 8 } & $\begin{array}{c}\text { COMP } \\
\text { EXTRA }\end{array}$ & VEN & 0 & 1 & 2 & 3 & 4 & 5 & 6 & 7 & 8 & 9 & 10 \\
\hline
\end{tabular}

